\title{
Low-temperature specific heat and thermal conductivity of glycerol
}

\author{
C. Talón, Q. W. Zou, M. A. Ramos, R. Villar, and S. Vieira \\ Laboratorio de Bajas Temperaturas, Departamento de Física de la Materia Condensada, C-III \\ Instituto de Ciencia de Materiales "Nicolás Cabrera", \\ Universidad Autónoma de Madrid, Cantoblanco, E-28049 Madrid, Spain
}

(Dated: October 23, 2018)

\begin{abstract}
We have measured the thermal conductivity of glassy glycerol between $1.5 \mathrm{~K}$ and $100 \mathrm{~K}$, as well as the specific heat of both glassy and crystalline phases of glycerol between $0.5 \mathrm{~K}$ and $25 \mathrm{~K}$. We discuss both low-temperature properties of this typical molecular glass in terms of the soft-potential model. Our finding of an excellent agreement between its predictions and experimental data for these two independent measurements constitutes a robust proof of the capabilities of the soft-potential model to account for the low-temperature properties of glasses in a wide temperature range.
\end{abstract}

PACS numbers: PACS: $65.40 .+\mathrm{g}, 66.70 .+\mathrm{f}, 63.50 .+\mathrm{x}$

It is well established国, that glasses or amorphous solids exhibit thermal properties very different from those of crystalline solids and, even more strikingly, very similar among themselves irrespective of the type of material, chemical bonding, etc. At temperatures $T<1 \mathrm{~K}$, the specific heat $C_{p}$ of non-metallic glasses is significantly larger and the thermal conductivity $\kappa$ orders of magnitude lower than those found in their crystalline counterparts. $C_{p}$ depends approximately linearly $\left(C_{p} \propto T\right)$ and $\kappa$ almost quadratically $\left(\kappa \propto T^{2}\right)$ on temperature, in contrast to the cubic dependences observed in crystals for both properties, well understood in terms of Debye's theory. At $T>1 \mathrm{~K}, C_{p}$ still deviates strongly from the expected $C_{\text {Debye }} \propto T^{3}$ dependence, exhibiting a hump in $C_{p} / T^{3}$. In the same temperature range the thermal conductivity exhibits a universal plateau.

In 1972, Phillip 3 and Anderson, Halperin and Varmal introduced independently the well-known tunneling model (TM), whose fundamental postulate was the ubiquitous existence of small groups of atoms in amorphous solids which can tunnel between two configurations of very similar energy. This simple model of two-level systems or tunneling states successfully explained the low-temperature properties of amorphous solids 0 , though only for $T<1 \mathrm{~K}$. On the contrary, the also rich universal behavior of glasses above $1 \mathrm{~K}$ (the hump in $C_{p} / T^{3}$ and the plateau in the thermal conductivity, or the remarkable feature in the vibrational density of states $g(\nu) / \nu^{2}$ at low frequencies known as the boson peak) still remains a matter of debate. One of the best accepted approaches to understand all the general behavior of glasses in the whole range of lowenergy excitations is the phenomenological soft-potential model (SPM), which ann be regarded as an extension of the TM. The SPME postulates the coexistence of extended lattice vibrations (sound waves) with quasilocalized low-frequency (soft) modes. In this model, the potential of these soft modes has a uniform stabilizing fourth-order term $W$. In addition, each mode has its individual first-order asymmetry $D_{1}$ and second-order restoring force terms $D_{2}$, which can be either positive or negative. Similarly to the TM, a random distribution of potentials is assumed. $P\left(D_{1}, D_{2}\right)=P^{\text {The SPM has }}$

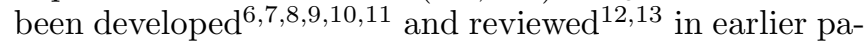
pers where the interested reader is referred to.

Glycerol $\left[\mathrm{C}_{3} \mathrm{H}_{5}(\mathrm{OH})_{3}\right]$ is probably the most widely studied 14 glass-forming liquid. Its high viscosity at a melting point around room temperature $\left(T_{m}=291 \mathrm{~K}\right)$ provides experimentalists with a very convenient temperature range where the supercooled liquid can be studied. Below the glass transition at $T_{g} \simeq 185 \mathrm{~K}$, the frozen-in liquid becomes a glass with a relatively weak, hydrogen-bonded network structure. Despite its good glass-forming ability, glycerol gan also be obtained in an orthorhombic crystalline state 25 , with four $\mathrm{C}_{3} \mathrm{H}_{5}(\mathrm{OH})_{3}$ molecules per unit cell, building up a structure of infinite hydrogen-bonded chains $1 \mathrm{~d}$. Several measurements of the specific teat fhis well-known glass have been indeed reported 17.18.19.20, though not reaching temperatures below $1.5 \mathrm{~K}$ in any gage. A broad maximum in $C_{p} / T^{3}$ was clearly observed 1920 around $8.5 \mathrm{~K}$, but the expected existence of tunneling states could not be determined, since it requires temperatures typically below $1 \mathrm{~K}$. Furthermore, Calemczuk et al. 0 also measured the specific heat of the crystalline state of glycerol, but only down to $5 \mathrm{~K}$, hence not reaching temperatures low enough as to assess its Debye coefficient. On the other hand, there are no published thermal-conductivity data at low temperatures neither for glycerol nor for any other similar molecular glass. This is very probably due to the experimental difficulties of adapting the thermal-conductivity technique to a sample which is liquid at ambient temperature and must be thermally controlled in situ to freeze it into the glass state, maintaining at the same time an appropriate geometry and a moderate heat flow for the thermal conductivity to be correctly measured. To our knowledge, only a few orientationally-disordered ("glassy") crustals from other molecular liquids have been measured 21 22.

In this work, we report thermal-conductivity data of glassy glycerol measured from $1.5 \mathrm{~K}$ to around $100 \mathrm{~K}$, as well as specific-heat measurements of both glassy and crystalline glycerol between $0.5 \mathrm{~K}$ and $25 \mathrm{~K}$. In addition, we make concurrent use of both thermal properties measured for the glassy state in order to test the validity of 
the soft-potential model in a typical molecular glass.

In Fig. 11(a), we show our thermal-conductivity data of glassy glycerol obtained by cooling in situ below $T_{g}$ at a rate around $-1 \mathrm{~K} / \mathrm{min}$ liquid glycerol (Merck, anhydrous, used without futher purification), placed inside a very thin-walled $(0.2 \mathrm{~mm})$ nylon tube. Standard steadystate techniques were employed. The thermal conductivity of the empty nylon tube was independently measured and subtracted. As can be seen, glycerol exhibits the thermal-conductivity behavior typical of glasses, with a plateau around $10 \mathrm{~K}$ quantitatively very similar to that of strong, network glasses as $\mathrm{As}_{2} \mathrm{~S}_{3}$ and $\mathrm{GeO}_{2}$ 2, i.e., a relatively high thermal conductivity among glasses. Therefore, glycerol could be a useful heat exchange medium at low temperatures when used as a glassy matrix.

Let us first analyze the thermal conductivity of glycerol yithin the soft-potential model. According to the $\mathrm{SPM} 8$, the inverse mean-free path of the phonons carrying out the heat is simply the sum of three contributions: the resonant scattering of the sound waves by either tunneling states or by soft vibrational modes, and the scattering by classical relaxational processes in the same asymmetric double-well potentials responsible of the tunneling states. From these premises, and inserting the corresponding SPM expressions for these three inverse mean-free paths, the thermal conductivity $\kappa$ was found to be 11,13

$$
\kappa=\frac{2 k_{\mathrm{B}}}{3 \pi \bar{C}}\left(\frac{W}{h}\right)^{2} F(z),
$$

where

$F(z)=\int_{0}^{\infty} d x \frac{x^{3} \mathrm{e}^{-x}}{\left(1-\mathrm{e}^{-x}\right)^{2}} \frac{z^{2}}{1.1 \tanh (x / 2)+0.7 z^{3 / 4}+x^{3} z^{3 / 8}}$

$\bar{C}$ is the usual (averaged over longitudinal and transverse acoustic modes) dimensionless constant of the TM, directly related to the universal plateau in the internal friction $Q^{-1}=(\pi / 2) C$, and $z=k_{\mathrm{B}} T / W$, where the energy $W$ is the aforementioned parameter of the SPM, which marks the crossover from the tunneling-states region at the lowest temperatures to the soft-mgdes region above it. Indeed, $W$ can be determined 11.13 from the position of the maximum $T_{\max }$ in a $\kappa / T$ versus $T$ plot:

$$
W \simeq 1.6 k_{\mathrm{B}} T_{\max }
$$

$T_{\max }$ therefore separates the low-temperature range where resonant scattering by tunneling states dominates $\left(\kappa \propto T^{2}\right)$ from higher temperatures $(\kappa \approx$ const $)$ where the soft modes are the main scatterers of acoustic phonons.

Fig. 1 shows that SPM eqs. (11-2) provide a good fit to thermal-conductivity data of glycerol from the lowest temperatures up to the end of the plateau around $30 \mathrm{~K}$ The two parameters, $W$ and $\bar{C}$ are easily obtained 11.43 from the $\kappa / T$ plot [Fig. [l(b)]: the maximum position gives $W=4.3 \mathrm{~K}$ and its height $\bar{C}=1.9 \times 10^{-4}$. The latter
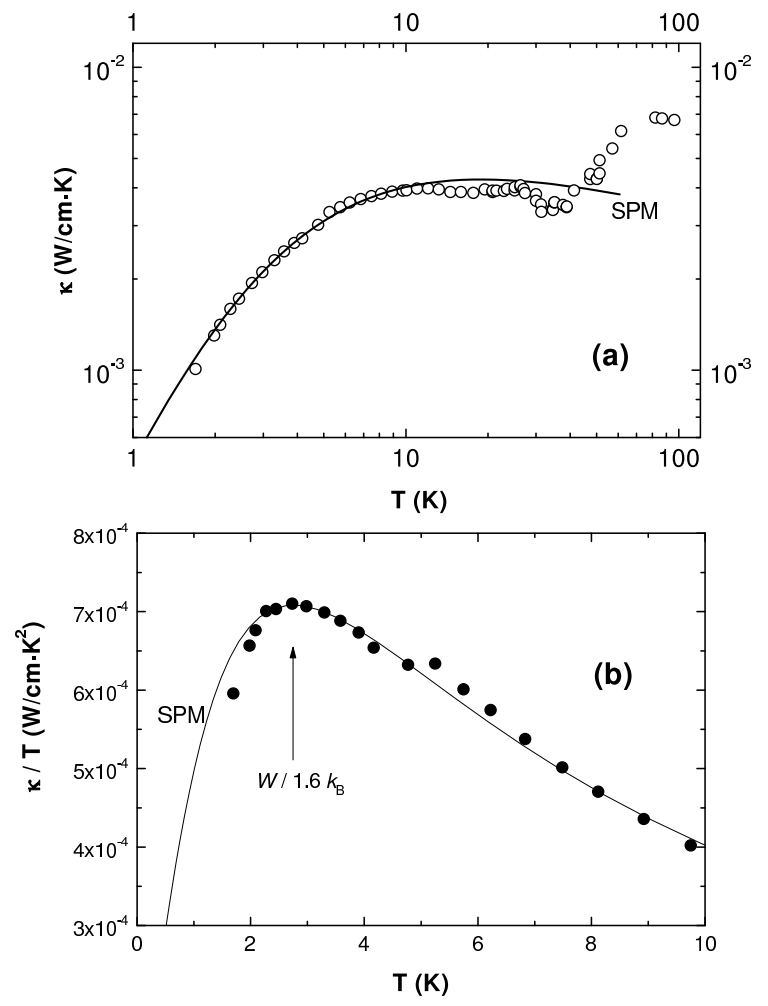

FIG. 1: Low-temperature thermal conductivity of glassy glycerol. (a): Thermal conductivity as a function of temperature in a log-log plot. Solid line is a fit to the soft-potential model (SPM). (b): Thermal conductivity divided by temperature below $10 \mathrm{~K}$. The maximum marks the crossover from tunneling states to quasilocalized vibrations (soft modes) as dominant scattering centers for the "phonons" and allows a direct determination of the SPM parameter $W$.

constitutes indeed a prediction for the low-temperature internal friction of glycerol, never measured to our knowledge: itsplateau value should be around $Q^{-1} \approx 3 \times 10^{-4}$, up to $1131.2 W / k_{\mathrm{B}} \simeq 5 \mathrm{~K}$, where the rise due to thermal relaxation should occur.

Our specific-heat measurements of glassy and crystalline states of glycerol are presented in Fig. 2 (in a log-log plot showing the whole low-temperature range) and in Fig. 3 (in a $C_{p} / T$ vs $T^{2}$ plot at the lowest temperatures to address the tunneling-states range for the glass and the Debye limit for the crystal). The heat capacity was measured in a ${ }^{3} \mathrm{He}$-cryostat, employing a lowtemperature quasi-adabiatic calorimetric cell, similar to one previously used in a ${ }^{4} \mathrm{He}-\mathrm{cryostat} 32 \mathrm{24}$. The liquid glycerol is placed in a vacuum-tight, thin-walled copper can, with a fine mesh of copper fitted inside to facilitate thermal equilibrium. A thin gold wire was used as heat switch to cool the experimental cell. The subtracted addenda contibution to the total measured heat capacity at $4.2 \mathrm{~K}(1 \mathrm{~K})$ was about $15 \%(22 \%)$ for the glass and about $30 \%(50 \%)$ for the crystal. The glass state was obtained by simply cooling the liquid from room temperature down to liquid-helium temperatures. After having 
measured its heat capacity at low temperature, glassy glycerol was slowly heated above $T_{g}$ (calorimetrically observed to take place at $185 \mathrm{~K}$ ) until it crystallized around 250-260 K. Once this first-order, exothermic transition was completed, the sample was cooled again and the heat capacity of the crystal measured.

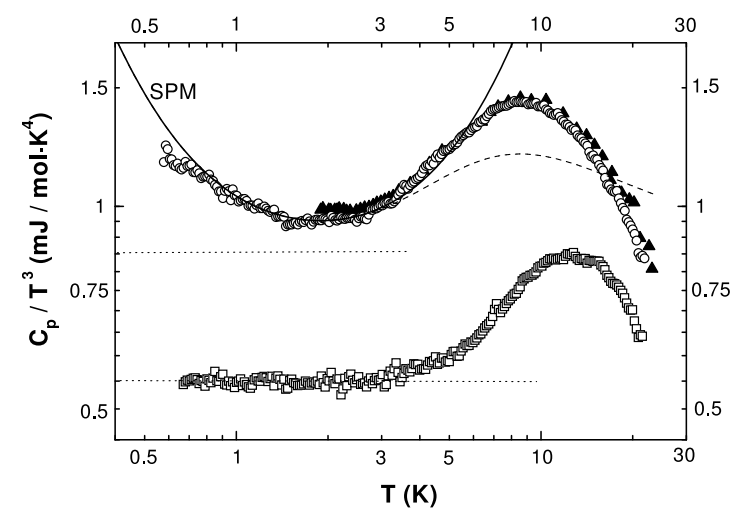

FIG. 2: Low-temperature specific heat $C_{p} / T^{3}$ of glass $(\bigcirc)$ and crystal $(\square)$ phases of glycerol for the whole measured temperature range in a log-log scale. Solid triangles are published data from Leadbetter and Wycherley 19 . Dotted lines show the correspondent Debye contributions to the specific heat either measured calorimetrically (crystal) or estimated from sound velocities (glass). Solid line shows the curve calculated with the soft-potential model (SPM), taking the parameter $W$ from the thermal conductivity data. Dashed line shows the same SPM calculation, but with a gaussian cutoff of the asymmetry for soft modes at high energies (see text).

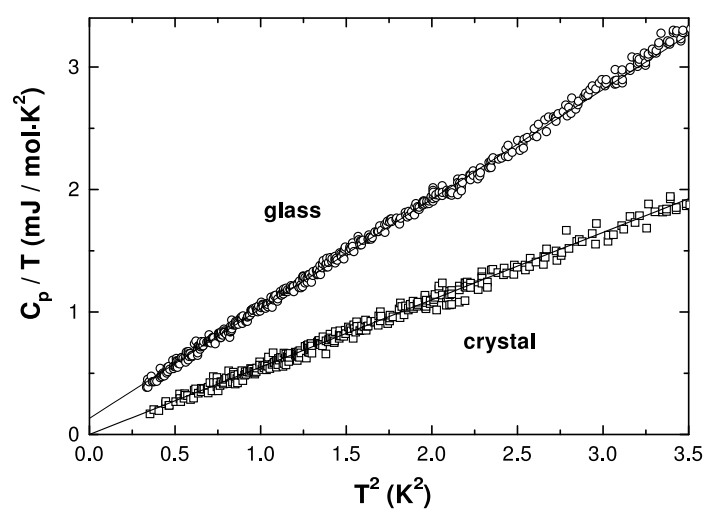

FIG. 3: Low-temperature specific heat of glass and crystal phases of glycerol in a $C_{p} / T$ vs $T^{2}$ plot. Symbols are as in Fig. 2. Solid lines are least-squares linear fits.

In Fig. 2, published data for the glass between 1.9 and $25 \mathrm{~K}$ from Leadbetter and Wycherley 19 can be seen to show a very good agreement with our data. There is also a good agreement with data for the crystal above $5 \mathrm{~K} 20$. The low-temperature cubic Debye contributions are also indicated in that figure: for the crystal, it has been obtained from a least-squares linear fit below $1.5 \mathrm{~K}$ in Fig. 3 , giving a Debye temperature $\Theta_{\mathrm{D}}=367 \mathrm{~K}$; for the glass, it can be estimated from zero-temperature extrapolations of elastic data25, 26, 27 to be $\Theta_{\mathrm{D}}=317 \mathrm{~K}$. In contrast to the crystal, where $C_{p}$ holds the expected Debye behavior for $T \leq \Theta_{\mathrm{D}} / 50$, the glass exhibits the typical excess over the Debye contribution in the whole low-temperature range. Within the SPM, these additional excitations are understood as soft quasiharmonic vibrations in single-well potentials responsible for the "boson peak" in $C_{p} / T^{3}$, together with tunneling states arising from related doublewell potentials responsible for the quasilinear contribution. The latter dominates below the minimum $T_{\min }$ in $C_{p} / T^{3}$ given by $W \approx 1.8-2 k_{\mathrm{B}} T_{\min } 12,13$, and is more clearly observed in Fig. 3, where a linear coefficient $\gamma=$ $0.13 \mathrm{~mJ} / \mathrm{mol} \cdot \mathrm{K}^{2}$ can be obtained (i.e., $\gamma=1.4 \mu \mathrm{J} / \mathrm{g} \cdot \mathrm{K}^{2}$ that is very similar to those values found in typical network glassest细).

Finally, we wish to compare the specific-heat data of glassy glycerol with the behavior predicted by the SPM, once we have previously obtained its basic parameter $W=4.3 \mathrm{~K}$ from thermal-conductivity data. As said above, the SPM postulates the coexistence of extended sound waves with quasilocalized modes, either tunneling states in double-well potentials or quasiharmonic vibrations in single-well potentials, with a gradual crossover between them characterized by the energy $W$. Therefore, we can write the specific heat for a glass as

$$
C_{p}=C_{\text {Debye }}+C_{\mathrm{TLS}}+C_{\mathrm{sm}},
$$

where $C_{\mathrm{sm}}$ is the contribution of soft modes (see below), and the contribution of the tunneling states $C_{\mathrm{TLS}}$ is determined, as in the TM, by means of the well-known expression for two-level systems $\mathrm{l}$, using the correspondent density-f tunneling states in terms of the SPM (eq. (4.7) in Ref.12 or eq. (9.30) in Ref.13).

In the standard SPM, the density of quasiharmonic soft vibrations increases continuously with frequency as

$$
g_{\mathrm{sm}}(h \nu)=\frac{1}{8} \frac{P_{s}}{W}\left(\frac{h \nu}{W}\right)^{4},
$$

where $P_{s}$ is the distribution constant of soft potentials. In the harmonic approximation, this leads ta specificheat contribution from soft modes given by 13

$$
C_{\mathrm{sm}}=\frac{2 \pi^{6}}{21} P_{s} k_{\mathrm{B}}\left(\frac{k_{\mathrm{B}} T}{W}\right)^{5} .
$$

The solid line in Fig. 2 shows the result of eq. (四), where $W=4.3 \mathrm{~K}$ was taken from the thermalconductivity measurement and $P_{s}=1.6 \times 10^{19} \mathrm{~mol}^{-1}$ was simply determined to scale with experimental data.

Obviously, the real distribution of soft modes cannot grow with frequency $g_{\mathrm{sm}}(\nu) \propto \nu^{4}$ unlimitedly. Gil et al. proposed a gaussian distribution in the asymmetry of the soft potentials (hence multiplying eq. (5) by an integral factor, see eq. (9.40) in Ref.13) based in a thermal strain ansatz, which without any further fitting parameter allowed them to account for the specific heat, thermal conductivity and vibrational density of states $g(\nu) / \nu^{2}$ in the 
whole relevant range, including the "boson peak". Alternatively, Gurevich et al.10 argued that the simple picture of independent quasilocalized harmonic vibrations coexisting with sound waves should fail at the Ioffe-Regel limit, not far above the boson peak. The interaction between soft modes would lead to a reconstruction of the vibrational density of states at higher frequencies, where delocalized soft vibrations with $g_{\mathrm{sm}}(\nu) \propto \nu$ should dominate, therefore also explaining the boson-peak feature. Furthermore, hybridization of acoustic phonons with quasilocalized modes has been proposed to set in around the boson peake8. For the sake of completeness, we also show in Fig. 2 by a dashed line the result of the total SPM predictionfor the specific heat if the abovementioned correction 93 of eq. (6) is used. As can be seen, the position of the maximum in $C_{p} / T^{3}$ (which only depends on $W$ and the glass transition temperature $T_{\mathrm{g}}$, hence being determined without any free parameter) is very well predicted, though its height is not so well accounted for. However, these quantitative agreements or disagreements of the SPM around or above the boson peak are perhaps not very relevant, since the low-energy limits of both independent quasilocalized modes and Debye acoustic phonons (note that even in the crystal, $C_{p}$ starts to deviate from the cubic limit above $5 \mathrm{~K}$ ) should begin to fail there, for the reasons mentioned above. Nevertheless, one may conclude that the maximum in $C_{p} / T^{3}$ of glasses (i.e., the boson peak) is just the fingerprint of the end for the low-energy distribution of independent soft modes, which govern low-temperature properties and low-frequency dynamics of glasses.

In any case, it is noteworthy that with only a constant factor $P_{s}$, used as free parameter to fit the height of the $C_{p}(T)$ curve ( $W$ was independently determined from thermal-conductivity data), the SPM is able to account consistently for the specific heat of this glass in the low-temperature range, from the tunneling-states region below $1 \mathrm{~K}$ up to the broad peak in $C_{p} / T^{3}$, including the crossover region around the minimum in $C_{p} / T^{3}$.

In summary, we have concurrently measured two lowtemperature thermal properties of a paradigmatic molecular glass, glycerol, as well as the specific heat of the crystalline phase between $0.5 \mathrm{~K}$ and $25 \mathrm{~K}$, hence being able to determine its Debye temperature. At lower temperatures, both properties for the glass exhibit a typical behavior indicative of the existence of tunneling states. Moreover, we have used these data as a new test of the SPM, which has been shown to successfully explain the specific heat and the thermal conductivity in a wide temperature range, also for a molecular glass such as glycerol.

\section{Acknowledgments}

This work was supported by MCyT (Spain) within project BFM2000-0035-C02-01.
1 R. C. Zeller and R. O. Pohl, Phys. Rev. B 4, 2029 (1971).

2 R. B. Stephens, Phys. Rev. B 8, 2896 (1973); Phys. Rev. B 13, 852 (1976).

3 W. A. Phillips, J. Low Temp. Phys. 7, 351 (1972).

${ }^{4}$ P. W. Anderson, B. I. Halperin, and C. M. Varma, Philos. Mag. 25, 1 (1972).

5 For a review, see: Amorphous Solids: Low Temperature Properties, edited by W. A. Phillips (Springer, Berlin, 1981); W. A. Phillips, Rep. Prog. Phys. 50, 1657 (1987).

6 V. G. Karpov, M. I. Klinger, and F. N. Ignat'ev, Zh. Eksp. Teor. Fiz. 84, 760 (1983) [Sov. Phys. JETP 57, 439 (1983)].

7 M. A. Il'in, V. G. Karpov, and D. A. Parshin, Zh. Eksp. Teor. Fiz. 92, 291 (1987) [Sov. Phys. JETP 65, 165 (1987)].

8 U. Buchenau, Yu. M. Galperin, V. L. Gurevich, D. A. Parshin, M. A. Ramos, and H. R. Schober, Phys. Rev. B 46, 2798 (1992).

9 L. Gil, M. A. Ramos, A. Bringer, and U. Buchenau, Phys. Rev. Lett. 70, 182 (1993).

10 V. L. Gurevich, D. A. Parshin, J. Pelous, and H. R. Schober, Phys. Rev. B 48, 16318 (1993).

11 M. A. Ramos and U. Buchenau, Phys. Rev. B 55, 5749 (1997).

12 D. A. Parshin, Phys. Rev. B 49, 9400 (1994).

13 M. A. Ramos and U. Buchenau, in Tunneling Systems in Amorphous and Crystalline Solids, edited by P. Esquinazi (Springer, Berlin, 1998), Chap. 9, pp. 527-569.

14 See, for instance, Refs. 1-43 of J. Wuttke, W. Petry, and S. Pouget, J. Chem. Phys. 105, 5177 (1996).
15 H. van Koningsveld, Rec. Trav. Chim. 87, 243 (1968).

16 F. J. Bermejo, A. Criado, A. de Andres, E. Enciso, and H. Schober, Phys. Rev. B 53, 5259 (1996).

17 J. E. Ahlberg, E. R. Blanchard, and W. O. Lundberg, J. Chem. Phys. 5, 539 (1937).

18 R. S. Craig, C. W. Massena, and R. M. Mallya, J. Appl. Phys. 36, 108 (1965).

19 A. J. Leadbetter and K. E. Wycherley, J. Chem. Thermodynamics 2, 855 (1970).

20 R. Calemczuk, R. Lagnier, and E. Bonjour, J. Non-Cryst. Solids 34, 149 (1979).

21 E. Bonjour, R. Calemczuk, R. Lagnier, and B. Salce, Journal de Physique 42, C6-63 (1981).

22 S. Vieira, M. A. Ramos, Q. W. Zou, and C. Talón, Phase Transitions 64, 87 (1997).

23 C. Talón, M. A. Ramos, S. Vieira, G. J. Cuello, F. J. Bermejo, A. Criado, M. L. Senent, S. M. Bennington, H. E. Fischer, and H. Schober, Phys. Rev. B 58, 745 (1998).

24 C. Talón, M. A. Ramos, S. Vieira, I. Shmyt'ko, N. Afonikova, A. Criado, G. Madariaga, and F. J. Bermejo, J. Non-Cryst. Solids 287, 226 (2001).

25 A. K. Schulz, J. de chim. phys. biol. 51, 324 (1954).

26 R. Piccirelli and T. Litovitz, J. Acoust. Soc. Am. 29, 1009 (1957).

27 Y. H. Jeong, S. R. Nagel, and S. Bhattacharya, Phys. Rev. A 34, 602 (1986).

28 S. I. Simdyankin, M. Dzugutov, S. N. Taraskin, and S. R. Elliott, Phys. Rev. B 63, 184301 (2001), and references 
therein. 